Proceedings

\title{
Influence of Buffer Thickness on Sensitivity of Pd-Coated Side Polished Single Mode Optical Fiber Hydrogen Sensor ${ }^{+}$
}

\author{
Mukesh P. Singh ${ }^{1, *}$ and Santosh Chaurasia ${ }^{2}$ \\ 1 Department of Applied Science \& Humanities, Jamia Millia Islamia, New Delhi 110025, India \\ 2 Department of Electrical \& Electronics Engineering, Academy of Business \& Engineering Sciences, \\ Ghaziabad 201009, India; santosh.chaurasia@abes.ac.in \\ * Correspondence: mpsingh@jmi.ac.in; Tel.: +91-986-893-3247 \\ † Presented at the Eurosensors 2018 Conference, Graz, Austria, 9-12 September 2018. \\ Published: 26 November 2018
}

\begin{abstract}
Optical fiber sensor which is based on palladium is highly sensitive to detect hydrogen. In this paper we analyzed the effect of buffer on sensitivity. Sensitivity is affected due to presence of buffer between side polished fiber core and hydrogen absorbing palladium layer. We optimized the buffer thicknesses for different palladium thickness to achieve maximum sensitivity.
\end{abstract}

Keywords: side polished fiber; buffer; hydrogen detection

\section{Introduction}

Consumption of traditional energy sources, i.e., hydrocarbons produces toxic gases which pollute the environment and have adverse effect on our health. The consumption of hydrogen as fuel do not produce any toxic material and hence it is being promised as one of the cleanest energy source [1]. Since gaseous volumetric hydrogen concentration in the range $4-74.5 \%$ in air (at room temperature and pressure) leads to an explosive atmosphere, highly sensitive sensors are required to monitor the presence of hydrogen in the environment [2]. Different types of semiconductor-based hydrogen sensors have been demonstrated [3]. These sensors exploit electrical characteristics for the detection purpose and hence may trigger an explosion during hydrogen leakage. In order to avoid this, optical fiber based sensors are preferred in hydrogenation environment because in this only optical signal is playing the role of sensing while its electrical parts, source and detector are located remotely, and also have a number of other advantages such as inherent safety, immunity to electromagnetic interference, and distributed remote sensing capability [4]. Different types of optical fiber hydrogen sensors are reported in literature and most of them are based on the interaction of the evanescent field with hydrogen through a transducer [5-7]. Recently Kim et al. have proposed a hydrogen sensor based on Pd coated side polished single mode fiber (SPSMF) [8]. In this design a single mode fiber is fixed in a quartz block and side polished to remove cladding to expose the evanescent tail of the field guided in the core of fiber. The polished face of the fiber is then coated with Pd which acts as a transducer.

\section{Numerical Procedure}

A schematic of SPSMF is shown in Figure 1 in which remaining cladding present between core and Pd layer is shown as buffer layer. 


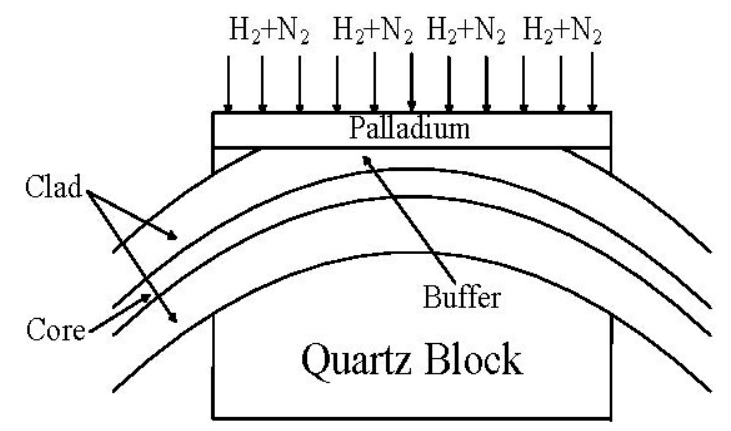

Figure 1. Schematic diagram of Pd coated side polished fiber half block (SMFHB) with remaining cladding (buffer) layer.

The equivalent planar model of this sensor is divided in three sections, namely input, sensor and output sections as shown in Figure 2. The input and output sections represent equivalent planar waveguide (EPWG) of the input and output of the single mode fibers and the polished side polished fiber half block (SMFHB) with buffer layer and Pd layer represented as sensor section. The modal indices and the field distribution of guided modes in all these sections were obtained using numerical procedure reported earlier [9]. At $z=0$, the excitation coefficients of the guided mode and surface plasmon wave (SPW) of sensing section and that of the guided mode of output section at $\mathrm{z}=\mathrm{L}$ as well as the power coupled to the sensing and output sections were obtained using the numerical procedure as suggested in Ref. [10]. The dielectric constant of Pd film changes during hydrogenation and hence the field distribution of SPW which leads to corresponding change in excitation coefficient and hence the power coupling to sensing and output sections. Since the optical power absorption decreases as the concentration of $\mathrm{H}_{2}$ in $\mathrm{Pd}$ increases, this change results in an increase in the optical power coupled into output section. The power coupling between the guided mode and SPW depends strongly on the thickness of the buffer layer, hence the thickness of remaining cladding, i.e., of the buffer layer is optimized to obtain maximized sensitivity of the sensor. The sensitivity of SMFHB sensor is defined as $S=10 \log _{10}\left(\mathrm{Pg}_{\mathrm{g}} / \mathrm{Pa}_{\mathrm{a}}\right)$ where $\mathrm{Pa}$ and $\mathrm{Pg}_{\mathrm{g}}$ are the output optical powers when the sensor section is exposed to pure $\mathrm{N}_{2}$ and mixture of $\mathrm{N}_{2}(96 \%)$ and $\mathrm{H}_{2}(4 \%)$ respectively. Since the SPW modes at dielectric/metal interface are supported only for TM modes, we have not considered TE modes in our calculations. The effect of radiation and reflected power at the input/sensor and sensor/output junctions are also neglected. All numerical simulations have been performed at wavelength $1550 \mathrm{~nm}$.

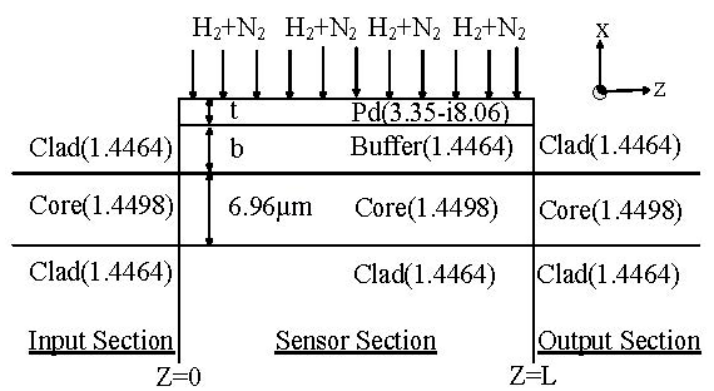

Figure 2. The equivalent planar waveguide model of side polished fiber with design parameters as used in Ref. [8].

\section{Result and Discussion}

When the palladium film absorbed the hydrogen, then complex dielectric constant of palladium film changes. The real and imaginary part of the dielectric constant increases almost linearly with increase in hydrogen concentration. When palladium becomes palladium hydride, its complex dielectric constant formulated by $\varepsilon p d(c)=h(c) \varepsilon p d(0)$, where the hydrogen constant $h(c)$ is 
the empirical decreasing function of the hydrogen concentration, $\mathrm{c}$ is the concentration of hydrogen in pd and $\varepsilon p d(0)$ is the complex dielectric constant of $\mathrm{Pd}$ in the absence of hydrogen [11].

Sensitivity as a function of buffer thickness for different transducer (Pd) thickness is shown in Figure 3.

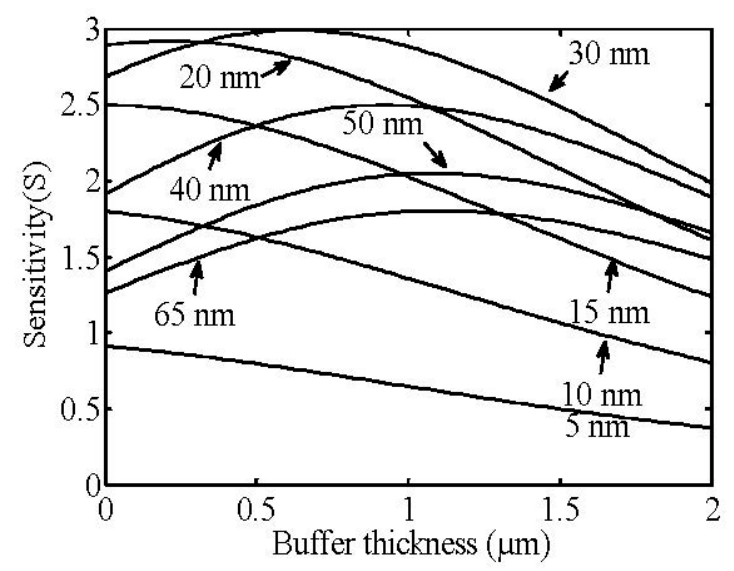

Figure 3. The sensitivity (S) of the SMFHB sensor as function of buffer thickness (b) for different palladium $(\mathrm{Pd})$ thickness.

The sensitivity shows a maxima for a given Pd thickness at certain buffer thickness. Figure 4 shows the variation of optimized buffer thickness and sensitivity as a function of palladium thickness. With the increase in Pd thickness the optimized buffer thickness increases initially showing a maximum value of $1.1 \mu \mathrm{m}$ for Pd thickness $56 \mathrm{~nm}$ and then gradually decreases and after that almost constant. Maximum sensitivity is obtained for Pd thickness $26 \mathrm{~nm}$ for optimized buffer thickness $0.62 \mu \mathrm{m}$. The sensitivity (S) of the sensor has been plotted as function of length of the sensor section in Figure 5 which exhibits that the sensitivity increases linearly with the increase in interaction length. With use of optimized buffer thickness an improvement of nearly $84 \%$ is found for the interaction length of $2.46 \mathrm{~mm}$ used in the experimental results reported in Ref. [8] in which the buffer thickness was $2 \mu \mathrm{m}$. Effect of hydrogen concentration in ppm (parts per million) on sensitivity for reported and optimized buffer and optimized transducer thickness is shown in Figure 6. Results show that sensitivity varies linearly with hydrogen concentration in transducer layer when the sensor is subjected to mixture of $\mathrm{N}_{2}(96 \%)$ and $\left(\mathrm{H}_{2} 4 \%\right)$ at partial pressure of about 630 torr at room temperature [12].

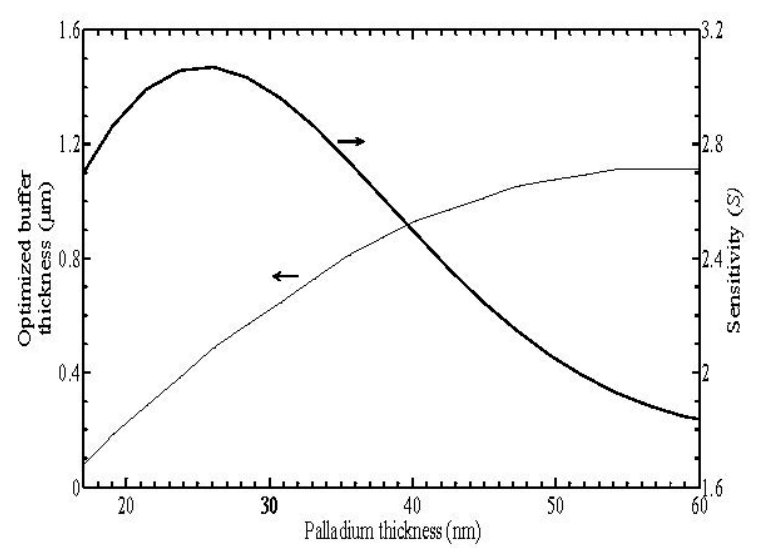

Figure 4. The optimized buffer thickness (- - ) and sensitivity (-) plotted as function of palladium layer thickness ( $\mathrm{t})$. 


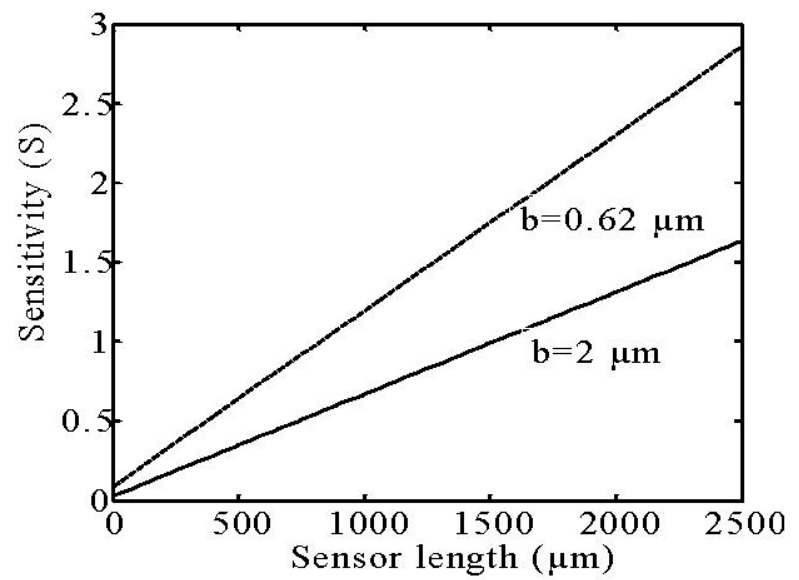

Figure 5. Sensitivity versus interaction length $(\mu \mathrm{m})$ of sensor with (dashed line) and without (solid line) optimized buffer thicknesses with Pd layer thickness of $20 \mathrm{~nm}$.

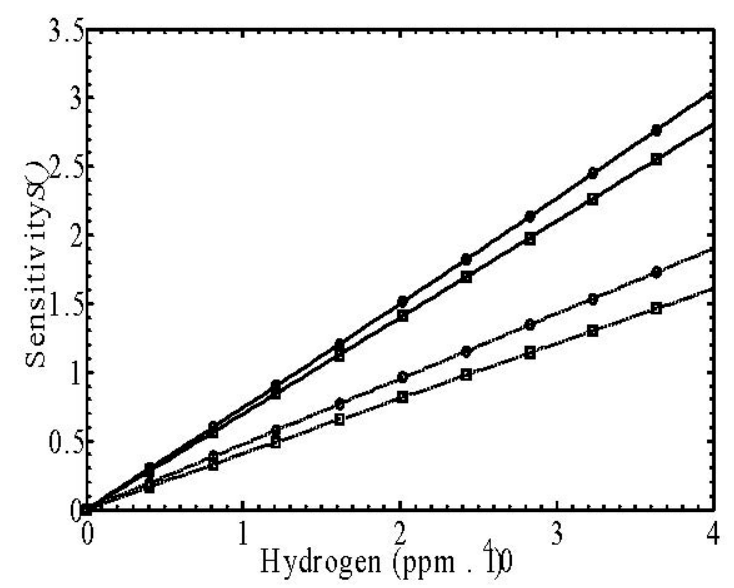

Figure 6. Sensitivity versus hydrogen concentration (ppm) in sensing layer for reported buffer thickness $b=2 \mu \mathrm{m}$ (---) optimized buffer thicknesses $b=0.62 \mu \mathrm{m}(-)$ with Pd layer thickness of $20 \mathrm{~nm}$ and optimized Pd layer thickness $26 \mathrm{~nm}$.

\section{Conclusions}

We have done the numerical investigation of single mode side polished fiber hydrogen sensor. The remaining thickness of cladding layer between the core and $\mathrm{Pd}$ acts as a buffer layer which controls the coupling between guided and plasmon modes. We studied this coupling effect and optimized thickness of this buffer layer to maximize the guided to plasmon mode coupling. We achieved an improvement of $84 \%$ in sensitivity with respect to the reported experimental results.

\section{References}

1. Töpler, J. Hydrogen as energy-storage-medium and fuel-A strong partner of renewable energies. Renew. Energy Environ. Sustain. 2016, 1, 31, doi:10.1051/rees/2016033.

2. Hübert, T.; Boon-Brett, L.; Black, G.; Banach, U. Hydrogen sensors-A review. Sens. Actuators B 2011, 157, 329-352, doi:10.1016/j.snb.2011.04.070

3. Christofides, C.; Mandelis, A. Solid-state sensor for trace hydrogen gas detection. J. Appl. Phys. 1990, 68, R1-R30, doi:10.1063/1.346398.

4. Orellana, G.; Haigh, D. New trends in fiber-optic chemical and biological sensors. Curr. Anal. Chem. 2008, 4, 273-295, doi:10.2174/157341108785914871.

5. Tabib-Azar, M.; Sutapun, B.; Petrick, R.; Kazemi, A. Highly sensitive hydrogen sensors using palladium coated fiber optics with exposed cores and evanescent field interactions. Sens. Actuators B 1999, 56, 158 163, doi:10.1016/j.snb.2005.05.010. 
6. Villatoro, J.; Díez, A.; Cruz, J.L.; Andrés, M.V. Highly sensitive optical hydrogen sensor using circular Pdcoated single-mode tapered fiber. Electron. Lett. 2001, 37, 1011-1012, doi:10.1016/j.snb.2005.05.010.

7. Zalvidea, D.; Díez, A.; Cruz, J.L.; Andrés, M.V. Hydrogen sensor based on a palladium-coated fibre-taper with improved time response. Sens. Actuators B 2006, 114, 268-274, doi:10.1016/j.snb.2005.05.010.

8. Kim, K.T.; Song, H.S.; Mah, J.P.; Hong, K.B.; Im, K.; Baik, S.; Yoon, Y.I. Hydrogen sensor based on palladium coated side-polished single-mode fiber. IEEE Sens. J. 2007, 7, 1767-1771, doi:10.1109/JSEN.2007.909924.

9. Sharma, E.K.; Singh, M.P. Multilayer waveguide devices with absorbing layers. J. Opt. Commun. 1993, 14, 134-137, doi:10.1515/JOC.1993.14.4.134.

10. Singh, M.P.; Sharma, A. Wavelength selectivity of in-line fiber optic filter devices. Appl. Opt. 1998, 37, 6350-6354, doi:10.1364/AO.37.006350.

11. Bevenot, X.; Trouillet, A.; Veillas, C.; Gagnaire, H.; Clement, M. Hydrogen leak detection using an optical fiber sensor for aerospace applications. Sens. Actuators B 2000, 67, 57-67, doi:10.1016/S0925-4005(00)00407-X.

12. Butler, M.A. Micromirror optical-fiber hydrogen sensor. Sens. Actuators B 1994, 22, 155-163, doi:10.1016/0925-4005(94)87015-2.

(C) 2018 by the authors. Licensee MDPI, Basel, Switzerland. This article is an open access article distributed under the terms and conditions of the Creative Commons Attribution (CC BY) license (http://creativecommons.org/licenses/by/4.0/). 